\title{
Resonances in Conductance Through Tunable Attractors
}

\author{
YONG S. JOE* and RONALD M. COSBY \\ Department of Physics and Astronomy, Ball State University, Muncie, IN 47306, USA
}

\begin{abstract}
We present the effects of resonant tunneling through doubly-coupled attractors in series in a quantum nanosystem. It is found that multiple split-tunneling peaks arise from resonant tunneling through multiple nondegenerate quasi-bound-states of the coupled attractors. We show that the tunability of coupling is achieved by modulating the strength of the attractor for a fixed separation length between the attractors, and by adjusting the geometrical separation length for a fixed intervening barrier strength.
\end{abstract}

Keywords: Nanostructures, Semiconductors, Tunneling

\section{INTRODUCTION}

Current state-of-the-art techniques result in surface-independent probes or contacts that may be biased to introduce tunable scattering potentials ("impurities") in quantum nanosystems [1]. Many theoretical and experimental works then have studied the elastic scattering by impurities in these systems. In particular, for a quantum structure containing a finite-size attractive impurity, called an 'attractor', it is slown that an attractor introduces multiple quasi-bound-states (QBSs) in the channel for a sufficiently strong attractor, and these states give rise to multiple resonant peaks before the first plateau in the conductance [2]. These conductance peaks appear due to resonant tunneling through the QBSs.
In this paper, we study the splitting of multiple resonant peaks before the first plateau in the conductance for a quantum nanosystem with two identical attractors in series. The separation of the split-conductance peaks through both the first and the second QBSs depends strongly on the coupling between the two attractors, which can be tuned by modulating the intervening barrier strength [3] and/or changing the geometrical separation length.

\section{MODEL AND METHOD}

A sketch of the model two-dimensional nanosystem with two identical attractors in series is illustrated in Figure 1(a), where relevant para-

*Corresponding author. Tel.: (765) 285-8879, Fax: (765) 265-5674, e-mail: ysjoe@bsu-cs.bsu.edu. 
(a)

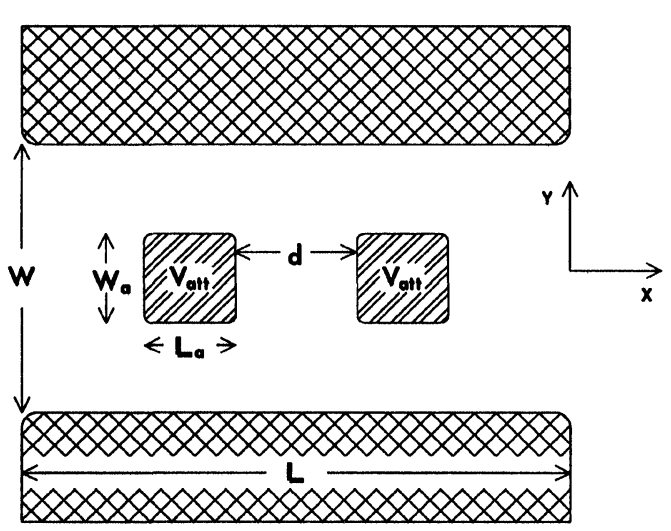

(b)

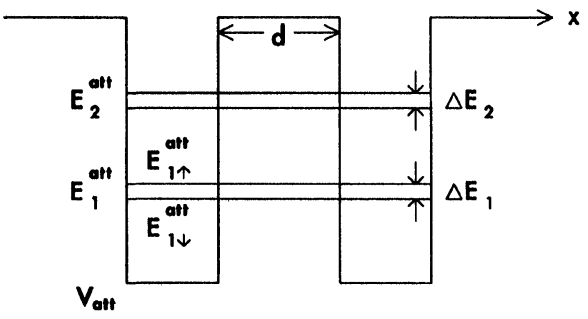

FIGURE 1 (a) Quantum structure studied: Two identical finite-size attractors are coupled with the coupling length $d$ along the transport direction. (b) A schematic potential profile of double attractors along the channel, where two-nondegenerate sets of QBSs are shown in a potential well.

meters are defined. We present results for the case where $W / W_{a}=2, W_{a} / L_{a}=1$, and the attractors are centered in the channel. We note that similar types of the structure modeled here, using several different techniques, have been realized experimentally [4]. For instance, Geim et al. [5] studied resonant tunneling through "donor pair-molecules" with random separation in quantum devices. Figure 1(b) illustrates a schematic potential profile of double attractors along the channel, where two-nondegenerate sets of QBSs are shown in a potential well.

Our calculations of the conductance are based on a nearest-neighbor tight-binding (TB) Hamiltonian and a recursive Green's function technique. Electrons in this system are free to move in the $x$ direction but are confined in the $y$-direction, as described by the Schrödinger equation $\left\{-\hbar^{2} / 2 m\right.$
$\left.\nabla_{y}^{2}+V_{c}(y)-E_{n}\right\} \psi_{n}(y)=0$, where $V_{c}(y)=\infty$ for the regions defined by hard walls [shown as cross-shaded regions in Figure 1(a)], and $V_{c}(y)=-V_{\text {att }}$ in the region with the attractors for $|2 y| \leq W_{a}$ [shown as shaded regions], and otherwise $V_{c}(y)=0$.

By discretizing the system spatially with lattice constant $a$, the resulting TB Hamiltonian is exactly solved by using the recursive Green's function method [6]. The Green's function $\mathbf{G}$ of a system in the presence of a perturbation $\hat{\mathbf{V}}$ is related to the Green's function $\mathbf{G}^{\circ}$ in the absence of $\hat{\mathbf{V}}$. The necessary recursion relations are obtained by taking the appropriate matrix elements of Dyson's equation, $\mathbf{G}=\mathbf{G}^{\circ}+\mathbf{G}^{\circ} \hat{\mathbf{V}} \mathbf{G}$. The conductance is then obtained using the Landauer equation [7], $G$ $=\left(2 e^{2} / h\right) \Sigma_{\mu \nu}\left|t_{\mu \nu}\right|^{2}=\left(2 e^{2} / h\right) \operatorname{Tr}\left(\mathbf{t t}^{\dagger}\right)$, where $\mathbf{t}$ is the transmission matrix.

\section{RESULTS AND DISCUSSIONS}

We investigate conductance peak splitting due to resonant tunneling through multiple QBSs in a quantum system with two attractors in series. Figure 2 shows the conductance $G$ of the structure as a function of the Fermi energy for different attractor strengths. (Here, the Fermi energy is

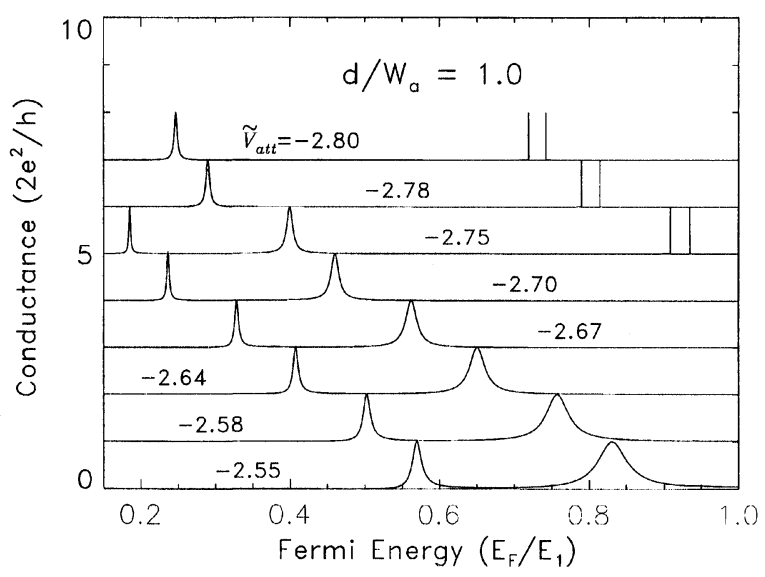

FIGURE 2 Splitting of conductance peaks before the first plateau as a function of the Fermi energy for different attractor strengths $\tilde{V}_{\text {att }}$ and a constant coupling length. 
normalized with respect to the first subband in the channel $\left.W, E_{1}=\hbar^{2} \pi^{2} / 2 m W^{2}\right)$. These curves are vertically offset one with respect to the other by one conductance unit for clarity. It is clearly seen that resonant tunneling peaks [two for $\tilde{V}_{\text {att }}$ $\left(=\sqrt{\left.V_{\text {att }} / E_{1}\right)}\right.$ in the range from -2.55 to -2.70 and four for $\tilde{V}_{\text {att }}$ from -2.75 to -2.80$]$ appear before the first plateau $\left(E_{F} / E_{1}=1\right)$ in the conductance. The appearance of these peaks is a consequence of both the formation of multiple QBSs in the attractor [2] [when an incident energy of the electron becomes the resonant energy $E_{F}^{\text {res }}$, the longitudinal momenta $p_{n, x}$ are enhanced because the QBSs $E_{n}^{\text {att }}$ have negative values $\left.\left(p_{n, x}=\sqrt{2 m\left(E_{F}^{\text {res }}-E_{n}^{\text {att }}\right)}\right)\right]$, and the overlap of these QBSs due to the coupling between the two attractors [3].

First, we note that the resonance widths of each peak through $E_{1}^{\text {att }}$ and $E_{2}^{\text {att }}$ are very different; a qualitative explanation follows. While $E_{F}$ varies from 0 to $E_{1}$, all subbands $E_{m}$ in the narrow channel without an impurity are evanescent modes. The mode coupling between $E_{m}$ and $E_{n}^{\text {att }}$ plays an important role in determining the interaction matrix element, which is a vital factor in the calculation of the transmission coefficient and the conductance. Intermode couplings between $E_{m}$ and $E_{n}^{\text {att }}$ for $(m, n)$ combinations with even and odd modes yield negligible contributions because the modes have opposite parities. The resonant tunneling through $E_{1}^{\text {att }}$ is mainly due to the coupling between $E_{1}$ and $E_{1}^{\text {att }}$. Similarly, the resonant peak through $E_{2}^{\text {att }}$ is due to the coupling between $E_{2}$ and $E_{2}^{\text {att }}$. However, since the evanescent mode $E_{2}$ is always energetically further away from the Fermi energy than $E_{1}$, the coupling between $E_{2}$ and $E_{2}^{\text {att }}$ is weaker than that between $E_{1}$ and $E_{1}^{\text {att }}$. Therefore, when an electron moves from $E_{2}$ to the $E_{2}^{\text {att }}$ state, it stays a longer time in $E_{2}^{\text {att }}$ and barely tunnels through it because of weak coupling. This indicates that the mean lifetime is larger and the width of the tunneling peak is narrower.

Second, we discuss the splitting of tunneling peaks in the presence of coupling between attractors. For two identical series attractors, the single resonant peak through the first QBS of $E_{1}^{\text {att }}$ splits into two well-separated peaks, shown in Figure 2 in the lower range of the Fermi energy for most values of $\tilde{V}_{\text {att }}$ displayed. [Here, the first half of the split peaks is not shown for $\tilde{V}_{\text {att }}=-2.78$ and -2.80$]$. In addition to these peaks, a conductance peak through the second QBS of $E_{2}^{\text {att }}$ also splits into two sharp spikes for $\tilde{V}_{\text {att }}=$ $-2.75,-2.78$ and -2.80 . A more careful examination shows that each tunneling peak through $E_{2}^{\text {att }}$ has a Lorentzian shape centered around the resonance energy [2]. We attribute this peak splitting to the elimination of the energy degeneracy due to the coupling between two attractors. In other words, if the separation between identical attractors is small enough, then the aligned, uncoupled QBSs in each attractor overlap and produce new nondegenerate states of the coupled attractor system. For a simple two-attractor model, one coupled state is slightly higher $\left(E_{n, \uparrow}^{\text {att }}\right)$ and the other slightly below $\left(E_{n, \downarrow}^{\text {att }}\right)$ the uncoupled energy $\left(E_{n}^{\text {att }}\right)$ [see Fig. 1(b)], with a perfect tunneling rate indicated (i.e., transmittance through these states reaches one). Therefore, multiple split-conductance peaks arise from the resonant tunneling through multiple nondegenerate QBSs of the coupled attractors.

Next, we discuss the effect of the coupling between the attractors on conductance peak splitting. It is expected that the separation of the split-tunneling peaks in conductance is reduced, as the coupling between two attractors becomes weaker. This result is observed in the double and triple quantum dots, where a coupling (i.e., tunnel barrier) between the dots is controlled by separate gates [8]. Here, we show that the tunability of coupling can also be achieved by modulating (1) the strength of the attractor $\tilde{V}_{\text {att }}$ for a fixed separation length between the attractors, and (2) the coupling length $d$ (i.e., geometrical separation) for a fixed intervening barrier strength.

As the attractor depth $\left|\tilde{V}_{\text {att }}\right|$ increases, in Figure 2 , the separation of the split peaks through both the first and the second QBS becomes smaller. This is clearly seen in Figure 3, where the energy 


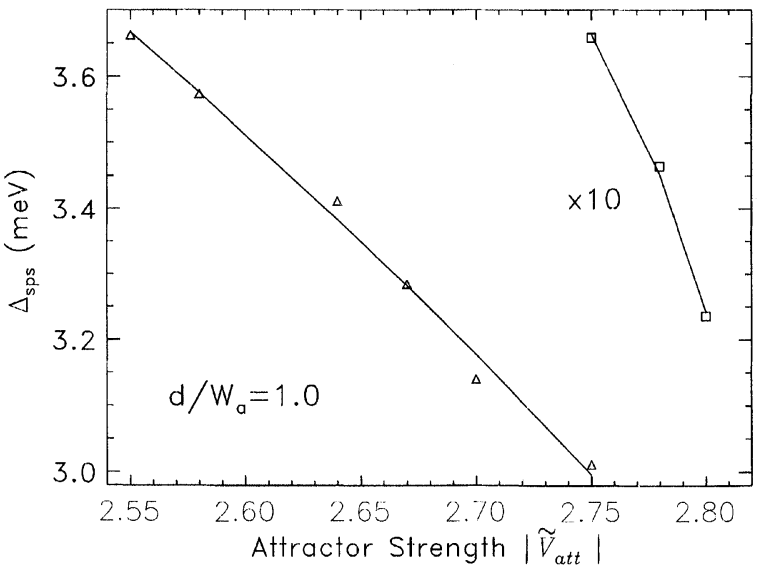

FIGURE 3 The separation of peak splitting $\Delta_{\text {sps }}$ as a function of $\left|\tilde{V}_{\text {att }}\right|$ is plotted, for a fixed coupling length $d /$ $W_{a}=1.0$, indicating that weak coupling between the attractors can be achieved by increasing $\tilde{V}_{\text {att }}$ resulting in strong localized QBSs in the attractor.

separation of peak splitting, $\Delta_{\text {sps }}$, as a function of $\left|\tilde{V}_{\text {att }}\right|$ is plotted. Here, $\Delta_{\text {sps }}$ through both nondegenerate states $E_{1}^{\text {att }}$ (triangles) and $E_{2}^{\text {att }}$ (squares; multiplied by a factor of 10 for clarity) decreases, albeit with different slopes. This can be explained by the fact that for a constant geometrical coupling between the attractors, a larger $\left|\tilde{V}_{\text {att }}\right|$ makes the QBSs lower in energy and more localized in the attractor, and therefore results in less interactions or overlaps between them. As a consequence, the separation of peak splitting in conductance decreases as $\left|\tilde{V}_{\text {att }}\right|$ increases. We expect that the peak splitting will physically disappear when $\left|\tilde{V}_{\text {att }}\right|$ is large enough to produce strong localized QBSs in each attractor, i.e., for an extremely weak coupling case.

Figure 4 shows the calculated conductance as a function of the Fermi energy for various coupling lengths. In order to see the effect of the geometrical coupling between the attractors on tunneling peak splitting, we focus on doubly split-tunneling peaks through the first two-nondegenerate QBSs $\left(E_{1, \uparrow}^{\text {att }}\right.$ and $\left.E_{1, \downarrow}^{\text {att }}\right)$ for a fixed $\tilde{V}_{\text {att }}=-2.75$. The geometrical separation length varies from $d / W_{a}=0.5$ (bottom curve) to $d / W_{a}=3.0$ (top curve) in steps of 0.1 , and the origin of the curve for different coupling length is shifted vertically in order to make a clear

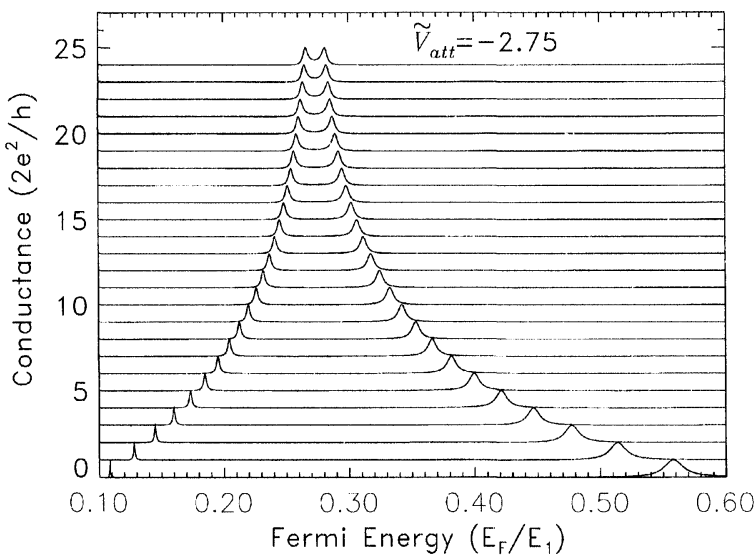

FIGURE 4 The doubly split-conductance peaks through the first two-nondegenerate QBSs for different coupling length. The geometrical separation length varies from $d / W_{a}=0.5$ (bottom curve) to $d / W_{a}=3.0$ (top curve) in steps of 0.1 , and the curves are vertically shifted for clarity.

comparison. As $d / W_{a}$ increases (i.e., the attractors are weakly coupled), the level separation $\Delta E_{1}$ $\left(=E_{1, \uparrow}^{\text {att }}-E_{1, \downarrow}^{\text {att }}\right)$ becomes narrower and hence the separation of the split-conductance peaks through these states is reduced in Figure 4. It is expected that when the attractors are far away from each other, each one has its original QBS $E_{1}^{\text {att }}$, and these degenerate states give rise to a single resonant peak before the first plateau. Notice that as $d / W_{a}$ increases, the width of the peak through $E_{1, \uparrow}^{\text {att }}$ becomes narrower because the QBS $E_{1, \uparrow}^{\text {att }}$ is more bound and localized around the attractor. As expected, this feature can be seen in the opposite way through the QBS $E_{1, \downarrow}^{\text {att }}$.

Figure 5 shows how the separation of the split peaks changes with increasing coupling length $d / W_{a}$. It appears that the energy separation of peak splitting, $\Delta_{\text {sps }}$, decreases exponentially as coupling length $d / W_{a}$ increases for $\tilde{V}_{\text {att }}=-2.75$. Therefore, for the split-conductance peaks through both $E_{1}^{\text {att }}$ and $E_{2}^{\text {att }}$, the coupling length $d$, in general, is related to $\Delta_{\text {sps }}$ as $d \approx-W \ln \left(\Delta_{\text {sps }}\right)$ for different $\tilde{V}_{\text {att }}$.

\section{CONCLUSIONS}

In summary, we have studied the effects of resonant tunneling through doubly-coupled at- 


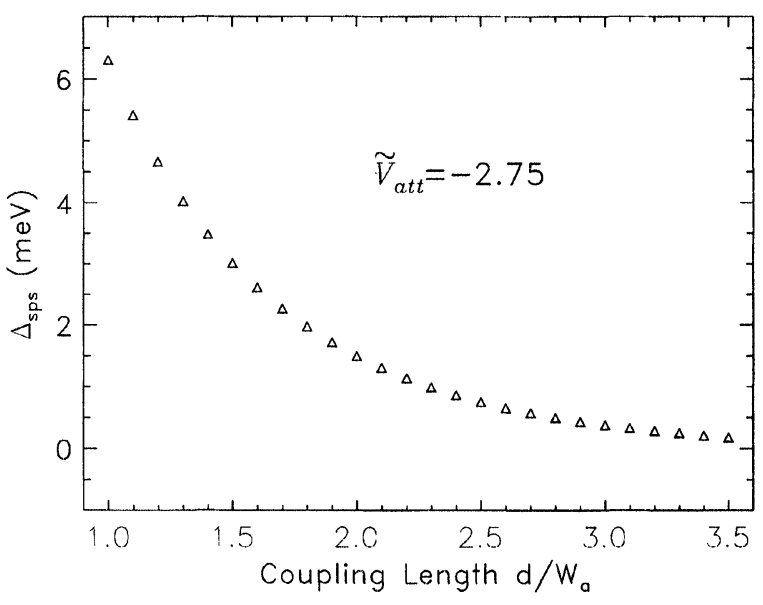

FIGURE 5 A relationship between the separation of the split peaks $\Delta_{\text {sps }}$ and coupling length $d / W_{a}$, showing that $\Delta_{\text {sps }}$ decreases exponentially as $d / W_{a}$ increases for $\tilde{V}_{\text {att }}=-2.75$.

tractors in series in a quantum nanosystem. We have found that the doubly split-conductance peaks arise from the resonant tunneling through two nondegenerate QBSs of the coupled attractörs, and the separation of the split-tunneling peaks are strongly affected by the coupling length and the intervening barrier strength.

\section{Acknowledgements}

This work was partially supported by the National Center for Supercomputing Applications under Grant Number DMR930007N and utilized the Convex Exemplar at the University of Illinois at Urbana-Champaign. RMC acknowledges partial support from the Center for Energy Research/ Education/Service at Ball State University.

\section{References}

[1] Feng, Y., Sachrajda, A. S., Taylor, R. P., Adams, J. A., Davies, M., Zawadzki, P., Coleridge, P. T., Landheer, D., Marshall, P. A. and Barber, R. (1993) Appl. Phys. Lett., 63, 1666.

[2] Joe, Y. S. and Cosby, R. M. (1997) J. Appl. Phys., 81, 6217.

[3] Joe, Y. S. and Cosby, R. M. (1997) Solid State Commun., $101,731$.

[4] Yamada, S. and Yamamoto, M. (1996) J. Appl. Phys., 79, 8391; Savchenko, A. K., Kuznetsov, V. V., Woolfe, A., Mace, D. R., Pepper, M., Ritchie, D. A. and Jones, G. A. C. (1995) Phys. Rev. B., 52, R17021; Eugster, C. C., del Alamo, J. A., Melloch, M. R. and Rooks, M. J. (1992) Phys. Rev. B., 46, 10146; McEuen, P. L., Alphenaar, B. W., Wheeler, R. G. and Sacks, R. N. (1990) Surf. Sci., 229, 312; Faist, J., Guéret, P. and Rothuizen, H. (1990) Phys. Rev. B., 42, 3217.

[5] Geim, A. K., Foster, T. J., Nogaret, A., Mori, N., McDonnell, P. J., La Scala, N. Jr., Main, P. C. and Eaves, L. (1994) Phys. Rev. B., 50, 8074.

[6] Joe, Y. S., Khatun, M. and Cosby, R. M. (1995) J. Appl. Phys., 78, 7120; Sols, F., Macucci, M., Ravaioli, U. and Hess, K. (1989) J. Appl. Phys., 66, 3892.

[7] Landauer, R. (1989) J. Phys. C., 1, 8099.

[8] Waugh, F. R., Berry, M. J., Mar, D. J., Westervelt, R. M., Campman, K. L. and Gossard, A. C. (1995) Phys. Rev. Lett., 75, 705; (1996) Phys. Rev. B., 53, 1413.

\section{Authors' Biographies}

Young S. Joe received his $\mathrm{Ph}$. D. in Condensed Matter Physics in 1992 from Ohio University, Athens, OH. Following an appointment as a Visiting Scholar at the National Research Council of Canada, he joined the faculty in the Department of Physics and Astronomy at Ball State University in 1993.

Ronald M. Cosby earned the $\mathrm{Ph}$. D. in Solid State Physics at the University of Kentucky in 1971 and holds masters' degrees in physics and computer science. He is a professor of physics at Ball State University, having served on the faculty of the Department of Physics and Astronomy since 1970. 

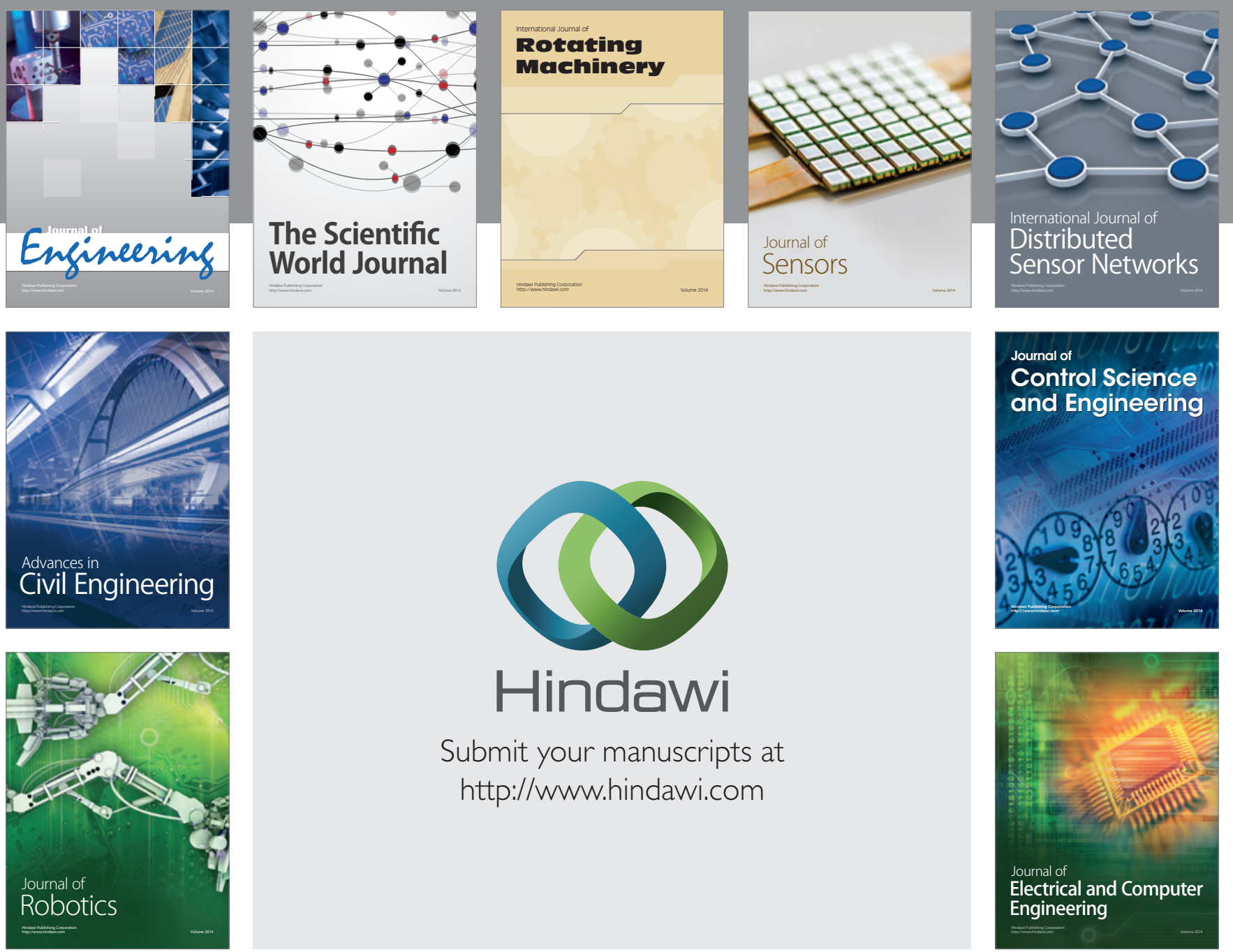

Submit your manuscripts at

http://www.hindawi.com
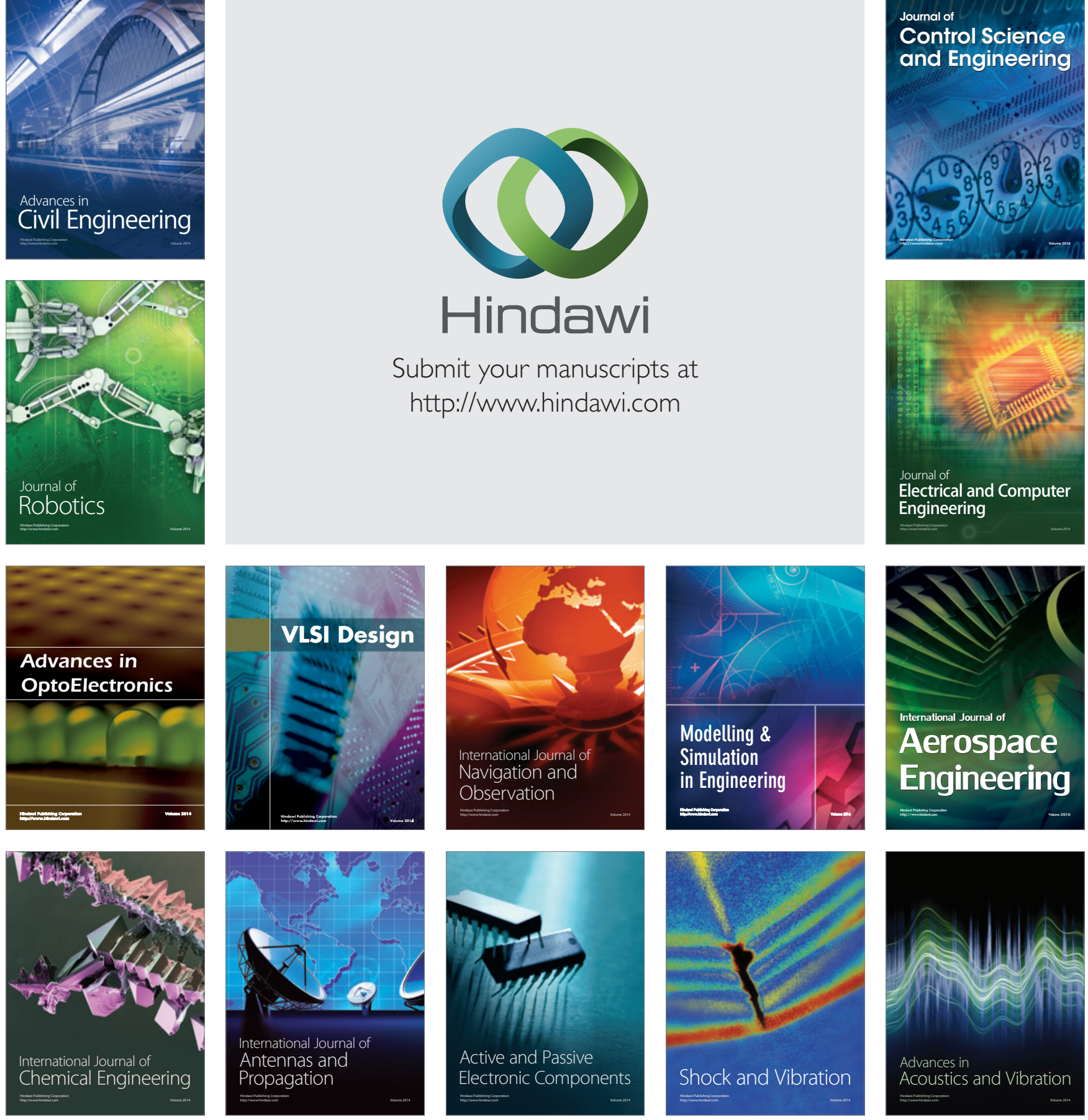